\title{
Pseudoangiomatous stromal hyperplasia: An observation on its microscopic involvement in breast carcinoma and the presence of lymph node metastases
}

\author{
JOHN D. COYNE \\ Department of Pathology, Royal Liverpool Hospital, Liverpool L7 8XP, UK
}

Received April 16, 2010; Accepted June 18, 2010

DOI: $10.3892 /$ ol_00000141

\begin{abstract}
The spaces of pseudoangiomatous stromal hyperplasia (PASH) are postulated to be important in the intramammary spread of breast carcinoma. The present study aimed to note the prevalence of inconspicuous, microscopic foci of PASH (identified as CD34+ve, CD31-ve and D2-40-ve spaces containing tumour emboli) involved in breast carcinoma and to establish the significance of its relationship to lymph node metastases. A total of 80 cases of breast carcinoma were examined for microscopic foci of PASH permeated by carcinoma and, of the four cases found to demonstrate such involvement, three had lymph node metastases.
\end{abstract}

\section{Introduction}

In order to determine whether microscopic involvement of pseudoangiomatous stromal hyperplasia (PASH) by breast cancer is associated with lymph node metastases, 80 cases of breast carcinoma in different categories were examined for microscopic foci of PASH. Of the 80 cases, 4 contained microscopic foci of PASH permeated by carcinoma. The results support the premise that PASH is an underestimated pathway of tumour spread.

\section{Materials and methods}

A total of 80 cases of infiltrating breast carcinoma in four different categories were examined. The materials used in this study were archived consecutive cases (mastectomy or wide local excisions) obtained from the files of the Department of Pathology at the University Hospital of South Manchester and were selected based on histological type, with and without nodal involvement. Carcinomas with vascular invasion were

Correspondence to: Dr John D. Coyne, Department of Pathology, Royal Liverpool Hospital, Duncan Building, Prescot Street, Liverpool L7 8XP, UK

E-mail: johnnycoyne@doctors.org.uk

Key words: breast, pseudoangiomatous stromal hyperplasia, lymph node metastases excluded. The categories included 10 grade 2 infiltrating ductal carcinomas with lymph node metastases, 10 grade 2 without lymph node metastases, 10 grade 3 infiltrating ductal carcinomas with lymph node metastases and 10 grade 3 carcinomas without lymph node metastases, respectively. In addition, four groups of infiltrating lobular carcinoma grades 2 and 3, with and without lymph node metastases were examined. All of the slides from the cases were reviewed. Any cases showing evidence of vascular invasion were excluded and a block from each case was randomly selected for immunohistochemistry. The sections were stained with CD34 (Novocastra) and smooth muscle actin (Sigma) to confirm or negate the presence of PASH with involvement by carcinoma (Figs. 1 and 2). A total of 4 cases with foci showing such involvement were additionally stained with antibodies to podoplanin (AngioBio), D2-40 (Zymed) and CD31.

\section{Results}

Of the 80 cases, 4 ( 2 grade 2 and 2 grade 3 infiltrating ductal carcinomas) contained microscopic foci of PASH measuring up to one high-power field, confirmed immunohistochemically, which was permeated by carcinoma. None of the cases displayed foci of vascular invasion. The 4 cases were also stained with antibodies to podoplanin (AngioBio), D2-40 (Zymed) and CD31 (Dako), and the CD34+ve spaces were unstained with these lymphatic and vascular markers. Lymph node involvement was noted in 3 of the 4 cases (Table I).

\section{Discussion}

Pseudoangiomatous stromal hyperplasia consists of slitlike anastomosing spaces lined by flattened elongated myofibroblasts with small nuclei and scanty cytoplasm. The spaces are separated by hyalinised connective tissue, and the cells are negative for vascular endothelial markers, including factor VIII and CD31, and for the lymphatic endothelial marker D2-40, but are positive for CD34 and smooth muscle actin $(1,2)$. Vuitch et al described PASH as a form of stromal hyperplasia considered to be the result of artefactual disruption and separation of collagen fibres with resulting open inter-anastomosing spaces (3). Findings by Hartveit showed that the ultrastructure of attenuated lymphatic 


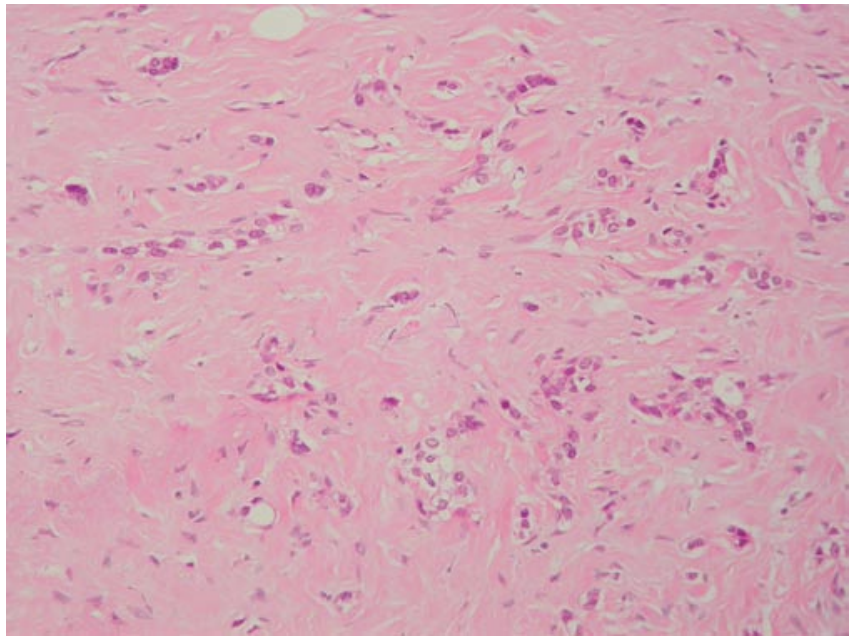

Figure 1. A barely conspicuous microscopic focus of pseudoangiomatous stromal hyperplasia involved by invasive carcinoma (H\&E).

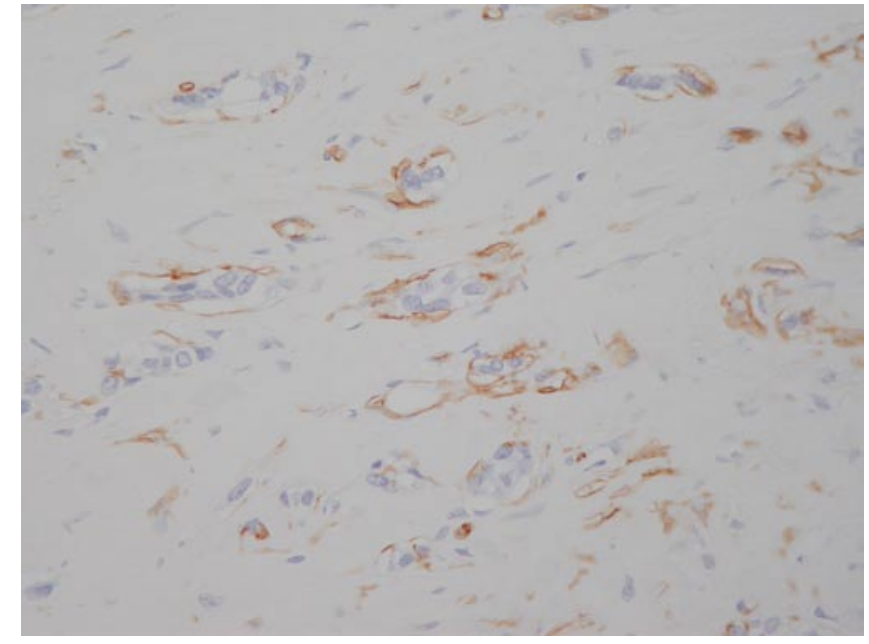

Figure 2. The same focus of invasive carcinoma within the microscopic spaces of pseudoangiomatous stromal hyperplasia highlighted by CD34 immunohistochemistry.

Table I. Pseudoangiomatous stromal hyperplasia; an observation on its presence in breast carcinoma and lymph node metastases.

\begin{tabular}{lccccr}
\hline Case no. & Age (years) & Carcinoma & Size $(\mathrm{mm})$ & Vascular invasion & Nodes \\
\hline 1 & 64 & Infiltrating ductal grade 2 & 18 & Not found & 1 of 13 \\
2 & 56 & Infiltrating ductal grade 3 & 15 & Not found & 4 of 15 \\
3 & 72 & Infiltrating ductal grade 3 & 25 & Not found & 1 of 24 \\
4 & 59 & Infiltrating ductal grade 2 & 10 & Not found & 0 of 17 \\
\hline
\end{tabular}

endothelial cells form sheets rather than vessels within the breast stroma and that these potential spaces form the missing lymphatic system of the breast, the lymphatic labyrinth $(4,5)$. Fisher et al concluded that PASH and this lymphatic labyrinth (spaces ultrastructurally lined by slender cells with tapering cytoplasmic processes that are either fibroblasts or lymphatic endothelial cells) are related structures (6). Recently, Asioli et al, in a three-dimensional study of two cases of normal breast tissue and one case of PASH, demonstrated direct anastomoses between pre-lymphatic channels and true lymphatics of the breast (2).

The missing lymphatic labyrinth described by Hartveit is now considered to be the normal counterpoint of the spaces that constitute pseudoangiomatous hyperplasia (7). Due to the fact that these channels communicate between breast epithelial/stromal structures and the main lymphatic system, it is also suggested that these pre-lymphatics should be considered in the intramammary spread of tumours, a suggestion previously posited by Damiani et al (1). These authors' observations, although not statistically valid, are supportive of this premise.

Axillary lymph node involvement is a powerful prognostic indicator (7). Undetected or unsampled lymphatic involvement in these 4 cases cannot be excluded, while the correlation of two findings does not necessarily establish a cause and effect relationship. However, the involvement of PASH may be a marker of such involvement, given the results of this study and the findings of Damiani et al (1) and Asioli et al (2). Furthermore, we cannot exclude such undetected or unsampled lymphatic involvement in other cases with lymph node metastases, but without vascular involvement and the absence of PASH foci. A recent study using antibodies to D2-40, podoplanin and Prox-1 concluded that lymphangiogenesis does not occur in breast cancer (8). Three additional studies documented the correlation between prominent separation/retraction artefact in breast cancer and lymph node metastases (10-12). One of these studies suggested that separation artefact may be early 'lymphovasculogenesis' prior to the mesenchymal cell being converted to the endothelial cell (10). An additional study postulates that retraction spaces are likely related to altered tumour-stromal interactions and are possibly an early stage of lymphatic tumour spread (12).

\section{References}

1. Damiani S, Peterse JL and Eusebi V: Malignant neoplasms infiltrating 'pseudoangiomatous' stromal hyperplasia of the breast: an unrecognised pathway of tumour spread. Histopathology 41: 208-215, 2002.

2. Asioli S, Eusebi V, Gaetano L, Losi L and Bussolati G: The prelymphatic pathway, the roots of the lymphatic system in breast tissue: a 3D study. Virchows Arch 453: 401-406, 2008.

3. Vuitch MF, Rosen PP and Erlandson RA: Pseudoangiomatous hyperplasia of mammary stroma. Hum Pathol 17: 185-191, 1986.

4. Hartveit F: Attenuated cells in breast stroma; the missing lymphatic system of the breast. Histopathology 16: 533-543, 1990.

5. Hartveit F: Pericytes in human breast stroma and the cells to which they relate. Eur J Morphol 30: 289-296, 1992. 
6. Fisher CJ, Hanby AM, Robinson L and Mills RR: Mammary hamartoma - a review of thirty-five cases. Histopathology 20 : 99-106, 1992.

7. Tavassoli FA and Eusebi V: Tumors of the Mammary Gland. AFIP Atlas of Tumor Pathology, 4th Series, Fasicle 10. ARP Press, Maryland, pp269-274, 2009.

8. Cserni G: Evaluation of sentinel lymph nodes in breast cancer. Histopathology 46: 697-702, 2005.

9. Agarmal B, Saxena R, Morimiya A, Mehrotra S and Badve S: Lymphoangiogenesis does not occur in breast cancer. Am J Surg Pathol 29: 1449-1455, 2005.
10. Barsky SH, Ye Y and Karlin NJ: 'Separation Artefact' V lymphovascular invasion: are mimics only mimics? Mod Pathol 86 (Suppl 1): 289A, 2006.

11. Acs G, Dumoff KL, Solin LJ, Pasha T, Xiaowei Xu and Zhang PJ: Extensive retraction artifact correlates with lymphatic and nodal metastasis and predicts poor outcome in early stage breast carcinoma. Am J Surg Pathol 31: 129-140, 2007.

12. Acs G, Paragh G, Shang-Tian C, Laronga C and Zhang PJ: The presence of micropapillary features and retraction artifact in core needle biopsy material predict lymph node metastasis in breast carcinoma. Am J Surg Pathol 33: 202-210, 2009. 